\title{
Cortical representation of the horizon in V1 and peripheral scaling in mammals with lateral eyes
}

\author{
Cristovam W. Picanço-Diniz ${ }^{1}$, Emiliana G. Rocha ${ }^{1}$, Luiz Carlos L. Silveira ${ }^{1}$, Guy Elston ${ }^{2}$ and \\ Eduardo Oswaldo-Cruz ${ }^{3}$ \\ 1 - Universidade Federal do Pará, Belém, PA, Brazil \\ 2 - Centre for Cognitive Neuroscience, Sunshine Coast, QLD, Australia \\ 3 - Universidade Federal do Rio de Janeiro, Rio de Janeiro, RJ, Brazil
}

\begin{abstract}
In the present investigation we mapped the primary visual area of the South American diurnal rodent, Dasyprocta aguti, by standardized electrophysiological mapping techniques. In particular, we performed a series of mapping experiments of the visual streak in the primary visual cortex. We found that the representation of the visual streak in V1 is greatly expanded, the nasal 10 degrees of the visual streak representation occupies ten times more cortical area than equivalent areas in the central or temporal representation. Comparison of these data with those on the density of ganglion cells in the retina at corresponding locations in the visual field reveal a significant mismatch between these two variables. The nasal representation is greatly expanded along the horizontal meridian in V1 as compared to the central and temporal regions whereas the density of ganglion cells decreases with progression along the visual streak from central region towards the nasal or temporal visual field. A review of the available data reveals that all lateral-eyed mammals exhibit a similar mismatch between the retinal and cortical representation of the visual field, and this mismatches is greater in those species with well defined visual streaks such as rabbit and agouti. KeyWords: horizontal meridian, V1 map, retinal ganglion cells, lateral-eyed mammals
\end{abstract}

Received 14 June 2011; received in revised form 29 June 2011; accepted 30 June 2011. Available on line 30 June 2011

\section{Introduction}

Previously we reported on the cytoarchitecture, myeloarchitecture and visuotopic representation of the primary visual area (V1 or area 17) in the South American diurnal rodent Dasyprocta aguti, and compared the visuotopic representation in V1 with ganglion cell density in the corresponding topographic location in the retina (Picanco-Diniz, Silveira, Carvalho, \&OswaldoCruz, 1991; Picanço-Diniz, Silveira, \&Oswaldo-Cruz, 1992). Of particular note was the prominent and asymmetric representation of the horizontal meridian

Cristovam Wanderley Picanço-Diniz, MD, PhD, Laboratory of Neurodegeneration and Infection, Federal University of Pará, Brazil. Emiliana G. Rocha, Universidade Federal do Pará, Belém, PA, Brazil. Luiz Carlos L. Silveira, Universidade Federal do Pará, Núcleo de Medicina Tropical, Belém, Brazil. Guy Elston, Centre for Cognitive Neuroscience, Sunshine Coast, Queensland 4562, Australia. and Eduardo Oswaldo-Cruz, Universidade Federal do Rio de Janeiro, Instituto de Biofísica Carlos Chagas Filho, Rio de Janeiro, Brazil. Correspondence regarding this article should be directed to: Cristovam Wanderley PicançoDiniz- Hospital Universitário João de Barros Barreto, Rua dos Mundurucus 4487, 66073-000 Belém/PA, Brazil. Phone: +55 91 3201-7741. E-mail: cwpdiniz@gmail.com in $\mathrm{V} 1$, corresponding to the elongated visual streak of the retina. As previously described to the rabbit (Hughes, 1971), asymmetries of the representation of the contralateral visual streak representation on agouti area 17 do not match the retinal ganglion-cell regional densities. On the other hand an expanded horizontal meridian has been reported in V1 of the guinea pig (Cavia porcellus), despite the poorly developed visual streak in this species (Choudhury, 1978). Thus, it remains unknown whether this apparent mismatch between a well developed visual streak in the retina and an expanded and asymmetric horizontal meridian representation in V1 in rodents reflect variation among species or result from different interpretation of early mapping studies. Here we remapped the cortical representation of the horizontal meridian in V1 of the agouti and reviewed the literature to add clarity to the data. We performed a series of electrophysiological mapping experiments, with individual electrode penetrations at closely spaced intervals, along the horizontal meridian in the agouti V1 and confirmed earlier reports of an expanded and asymmetric representation. Our comparative survey review indicates that a mismatch between the retinal and cortical representation of the nasal visual field is common in all mammals with laterally placed eyes. 


\section{Material and methods}

\section{Animals}

We used six adult male agoutis (Dasyprocta aguti), obtained from the Museu Paraense Emílio Göeldi animal colony in the present study. All efforts were made to avoid animal suffering and to reduce the number of specimens used. The use of agoutis was in accordance with guidelines from the Brazilian Institute of the Environment and Renewable Natural Resources (IBAMA) (license 207419-0030/2003). All experimental procedures were carried out strictly in accordance with the NIH Guide for the Care and Use of Laboratory Animals.

\section{Electrophysiological mapping}

Electrophysiological mapping techniques have been described in detail elsewhere (Picanco-Diniz et al., 1991). Briefly, the animals were anesthetized with a mixture of $5 \%$ ketamine chloridrate (Ketalar, ParkeDavis) and 2\% xylazine chloridrate (Rompum, Bayer) (i.m.). A stable anesthetic level was obtained using $1 \mathrm{ml}$ of the mixture/ $\mathrm{kg}$ of body weight at $2 \mathrm{~h}$ intervals.

Multiunit receptive fields were defined as the regions on the visual field where light stimulation, with hand-held cards drifted tangentially to the visual hemisphere, evoked changes in neuronal activity. Tungsten microelectrode and the differential amplifier were used to record action potentials from small clusters of neurons. When moving the electrode to an adjacent location in the cortex, a systematic shift in the corresponding receptive field location was encountered. The electrode was moved in $500 \mu \mathrm{m}$ steps mediolateral and anteroposterior. A complete topographic map was obtained with a large number of electrode penetrations exploring the visual field representation in such a way that the limits of the horizontal meridian cortical area $\left( \pm 10^{\circ}\right.$ lat $)$ were clearly defined.

What is apparent from the behavior of these animals is that the horizon is projected onto the visual streak in the retina when these animals are seated on their haunches eating food clutched in their front paws (Silveira et al., 1989). This head position in anestethized subjects was reproduced in the head holder orienting the line defined by the palpebral cleft at $25^{\circ}$ inclined in relation to the horizon (Figure 1). The animals secured in the stereotaxic holder had the right eye aligned with reference to an oculocentric equatorial azimuthal coordinate system. In this system, the vertical meridian roughly corresponded to the nasotemporal retinal decussation and the horizontal meridian corresponds to the visual streak (Silveira et al., 1989). Cycloplegia and mydriasis were obtained with $1 \%$ atropine sulfate applied to the eyes. The cornea was protected with a thin layer of silicone fluid (Dow Corning 200/350). The position of the optic disk on the visual field was checked throughout the experiment by means of a reversible ophthalmoscope.
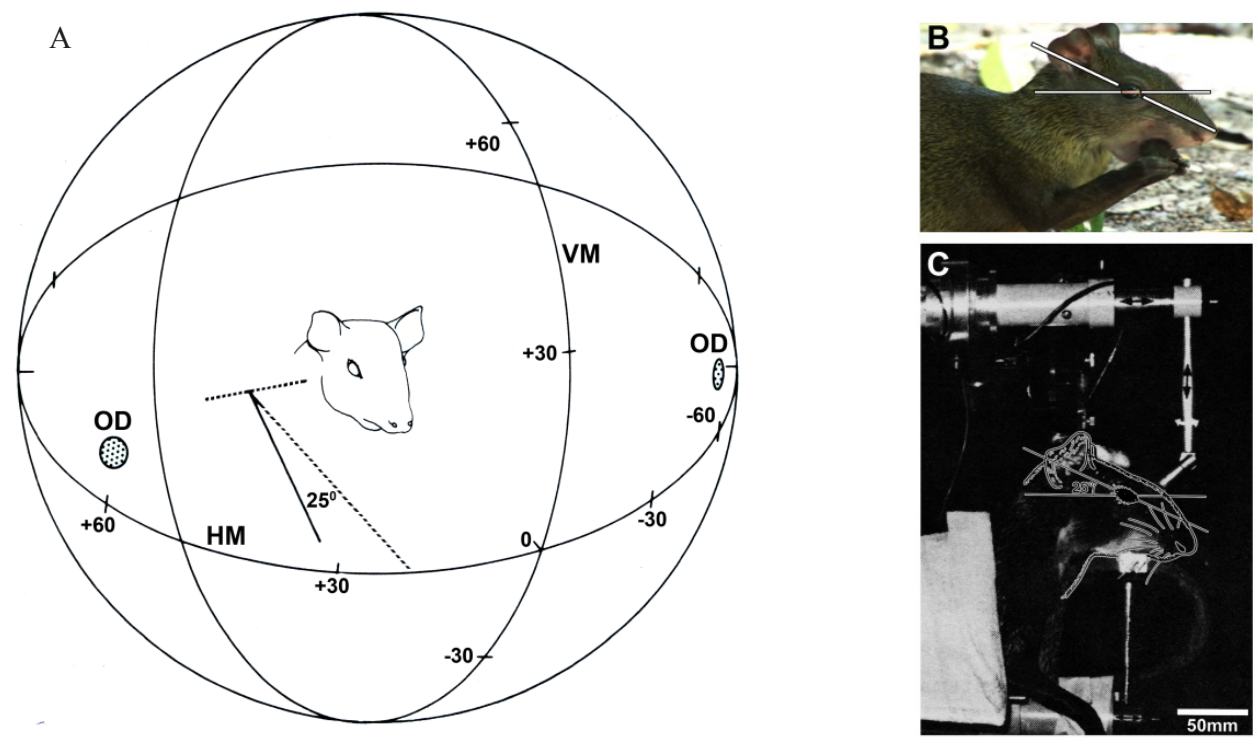

Figure 1. Relative position of the visual streak projection in the visual field. A: Diagrammatic representation of the relative position of the vertical meridian (VM), horizontal meridian (HM) and optic disks (OD). The eye was aligned with reference to an equatorial azimuthal coordinate system. A horizontal plane intersecting the corneal vertex of both eyes was made to coincide with the equatorial horizontal plane of a transparent hemisphere. The center of curvature of the hemisphere was made to coincide with the anterior nodal point of the right eye (Silveira, 1985). In this situation the parasagital plane containg the right eye's anterior nodal point corresponds to the zero-degree vertical meridian of the coordinate system. B: Picture of an agouti (Dasyprocta prymnolopha) in typical feeding posture. Note that agouti is a lateral-eyed mammal with a large monocular field. In typical feeding posture agouti's palpebral cleft present an angle of approximately $25^{\circ}$ with the horizontal plane and the visual streak is aligned to the horizon (Silveira, 1985). C: This head position in anestethized and paralyzed subjects was reproduced in the head holder allowing an unobstructed visual field. Ocular movements were prevented by intramuscular administration of neuromuscular blocker and the projection of the optic nerve head on the hemisphere was carried out with a reversible ophthalmoscope to monitor the eye position during the experiment. 
The electrocardiogram and electrocorticogram were recorded throughout the experiment and used to monitor the anesthetic level. Additional doses of anesthetic were given when necessary. Body core temperature was maintained at $37^{\circ} \mathrm{C}$. A tracheotomy was performed and the animal was paralyzed with $1 \mathrm{ml} / \mathrm{Kg}$ at 3 -h intervals of a solution of $2 \%$ trimethyl-gallamine (Flaxedil, Rhodia) diluted $1: 3$ in $0.9 \%$ saline solution and ventilated artificially. A craniotomy was performed, the dura-mater was reflected and the exposed cortical surface protected with warm mineral oil.

Perfusion, tissue preparation and anatomical reconstructions.

At the end of the electrophysiological recordings, the animals were deeply anaesthetised with a mixture of ketamine $(10 \mathrm{mg} / \mathrm{kg})$ and xylazine $(1 \mathrm{mg} / \mathrm{kg})$ (i.p.) and perfused transcardially with $0.9 \%$ heparinized- saline, followed by $4 \%$ paraformaldehyde (Sigma Company, USA) in $0.1 \mathrm{M}$ phosphate buffer (PB), $\mathrm{pH}$ 7.4. The brains were removed from the skull and processed so as to be able to align microelectrode tracks with gross anatomical landmarks, cytoarchitecture and myeloarchitecture. Sections were processed for Nissl substance to reveal cytoarchitecture and recognize the electrode trackings. All sections were mounted onto gelatinised-glass slices, air-dried overnight, dehydrated and coverslipped with Entellan (Merck). Electrode penetrations were correlated with anatomical landmarks by reconstructing across a series of sections, enlarged to allow easy visualization. A digitizing table was then used to measure the lateral position of each penetration in relation to the fundus of the lateral sulcus by generating a flat reconstruction of the visual recording sites.

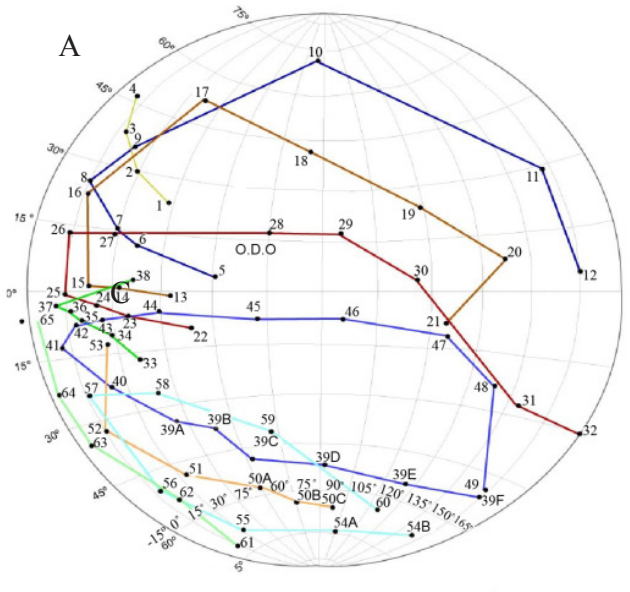

C

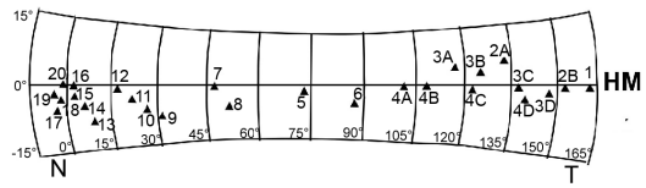

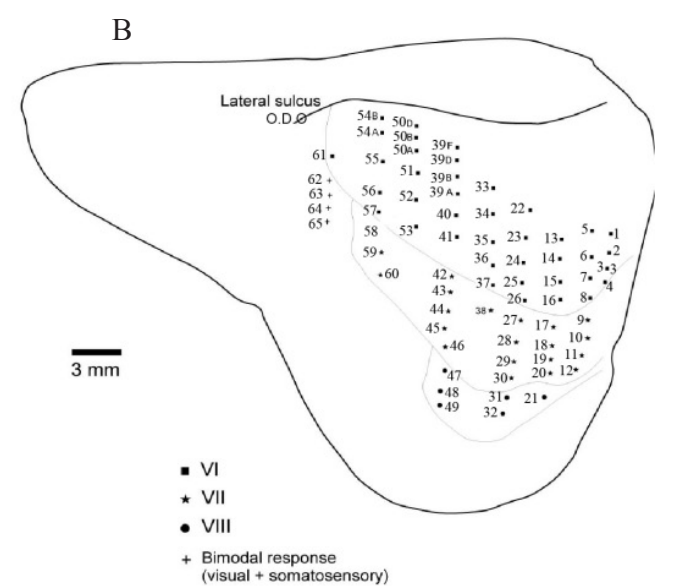

D
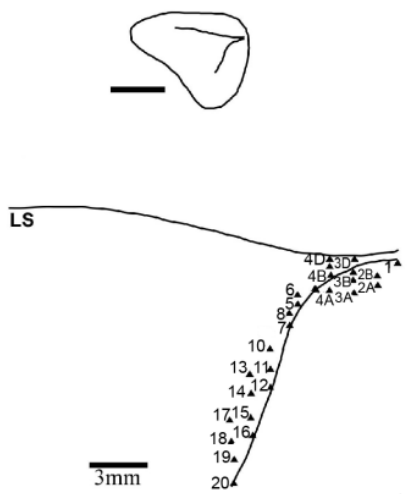

Figure 2. Mapping studies of V1 visual cortical area of agouti brain. Top: Graphic representation of the multiunit receptive field centers of visually responsive neurons and equivalent recording sites in the visual responsive cortex in the agouti brain (cases Da-830408 and Da-830122). Individual plots are numbered on the equatorial/azimuthal representation of the visual field (A) and the equivalent recording sites on the dorso-lateral cortical surface (B). Receptive field progressions along mediolateral transects from VI into the immediately adjacent second visual area V2 are depicted by different symbols. When the microelectrode was moved across the cortical surface in VI, the multiunit receptive field moved in orderly, continuous way through the visual field. Note in VI the lateral border represents the nasal border of the visual field, the rostral portion represents the lower field and the caudal region the upper field, as described previously (Picanço-Diniz, 1987; Picanço-Diniz et al., 1991). HM: Horizontal meridian; VM: Vertical meridian. Bottom: Graphic representation of the naso-temporal dimension of the visual streak in VI of case Da-830122. Small triangles indicate the center of the receptive fields on Equatorial/Azimuthal map of the visual field (C), and the equivalent cortical recording sites aligned with the representation of the horizontal meridian in VI (D). Numbers indicate cortical recording sites and equivalent receptive field centers. The inset indicates in a cortical dorso-lateral reconstruction the relative positions of the lateral sulcus and horizontal meridian representation. 


\section{Results}

Systematic electrophysiological mapping at closely spaced recording sites revealed an ordered topographic representation in the primary visual area of the agouti (Figure 2). In Figure 2 we have plotted the results of cases Da-830408 and Da-830122. On the equatorial/ azimuthal representation (A) is illustrated the center of multiunit receptive fields recorded along parallel sequences of mediolateral microelectrode penetrations in the primary visual area (V1). Vertical and horizontal meridians are represented as dotted lines in the lateral margin of V1 (Figure 2, B). Note that the receptive field position changes in an orderly way through the visual field when the microelectrode penetrations progressed laterally. As described previously (PicancoDiniz et al., 1991), there is a complete representation of the contralateral visual field in V1, where the lateral sulcus corresponds to the representation of the temporal periphery, whereas the lateral margin of V1 corresponds to the nasal end of the visual field. Lateral to the representation of the nasal end (vertical meridian of V1) is located V2 visual area. Note similar progressions along the nasotemporal dimension of the visual streak representation in case Da-830122 (Figure $2 \mathrm{C}$ and D).

The horizontal meridian, which runs roughly orthogonal to the lateral sulcus, occupies a disproportionately large portion of the complete visuotopic representation. In all cases we found that the visual streak representation in the visual field occupied $>40 \%$ of the entirety of V1. Moreover, we found visual field asymmetry along the nasotemporal axis of the horizontal meridian where the areal cortical magnification factor (ACMF) in the nasal region $\left(0.18 \mathrm{~mm}^{2} /\right.$ $\operatorname{deg}^{2}$; longitude $0^{\circ}$, latitude $0^{\circ}$ ) inside the representation of the binocular visual field is 10 times higher than that in the central region $\left(0.018 \mathrm{~mm}^{2} / \mathrm{deg}^{2}\right.$; longitude $68^{\circ}$, latitude $\left.0^{\circ}\right)$.

Figure 3 is a modification from case $\mathrm{Da} 830616$ (PicancoDiniz et al., 1991) to illustrate the mismatch between the visual streak nasotemporal distribution of retinal ganglion cells (Silveira, 1985) and areal cortical magnification factor (Picanço-Diniz, 1987) in the agouti V1 area. The quantitative analysis of the $\mathrm{V} 1$ map using the areal cortical magnification factor (ACMF) along the cortical representation of the visual streak demonstrates a significant nasotemporal asymmetry that cannot be directly associated with the retinal ganglion cell densities in the same region. In V1 the ACMF measured along the nasotemporal axis are highest in the region that corresponds to the intersection between the horizontal and vertical meridian and falls rapidly along the representation of the visual streak. In contrast, the retinal ganglion cell density shows a plateau along the visual streak decreasing sharply in the temporal and nasal extremities.

The binocular visual field mapping in the case Da850628 revealed that ACMF highest values are closer to the interception of the vertical and horizontal meridian at the nasal end of the visual streak representation (Figure 4).

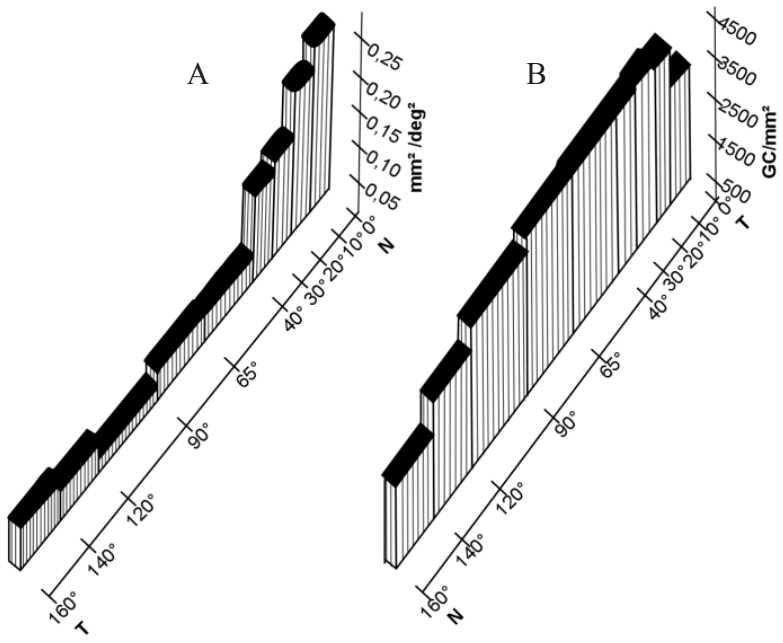

Figure 3. Areal cortical magnification factor (ACMF) along the visual streak representation in V1 versus ganglion cell densities in the agouti retina. Note the significant mismatch between ganglion cell topographical distribution and ACMF in the nasal end of the horizontal meridian in V1.

\section{Discussion}

The motivation for the present study were two-fold: to confirm the degree of expansion of the representation of the visual streak in V1 of the agouti and to establish whether there is a rational explanation for the apparent mismatch between the size of the nasal representation along the horizontal meridian in $\mathrm{V} 1$ and ganglion cell density along the visual streak in the retina. We found that the representation of the horizontal meridian is disproportionately large in this species. The central 20 degrees between the parallels \pm 10 in the visual field occupies $>40 \%$ of the visual representation in V1. Moreover, the expansion of the nasal portion of the horizontal meridian in V1 corresponds with a relatively low density of ganglion cells in this region in the visual streak in the retina.

A closer look at our mapping data reveal that the cortical representation of the contralateral visual streak in VI is asymmetric, the nasal field being disproportionately large when compared to the central (optic axis) and temporal fields (Picanco-Diniz et al., 1991).

Asymmetric representation of the contralateral visual field representation of the horizon in VI of lateral-eyed mammals

"Unsleeping eyes, by Nature raised

To take the horizon in..."

This description quoted by Austin Hughes in 1971 (Hughes, 1971) from an eighteenth century poet to describe the rabbit visual behavior can be expanded to all lateral-eyed mammals' with well defined visual streaks including the agouti. When the visual streak is aligned to the horizon this retinal specialization allows the animal to view the whole horizon without the specialized eye movements required for scanning with an area or fovea 

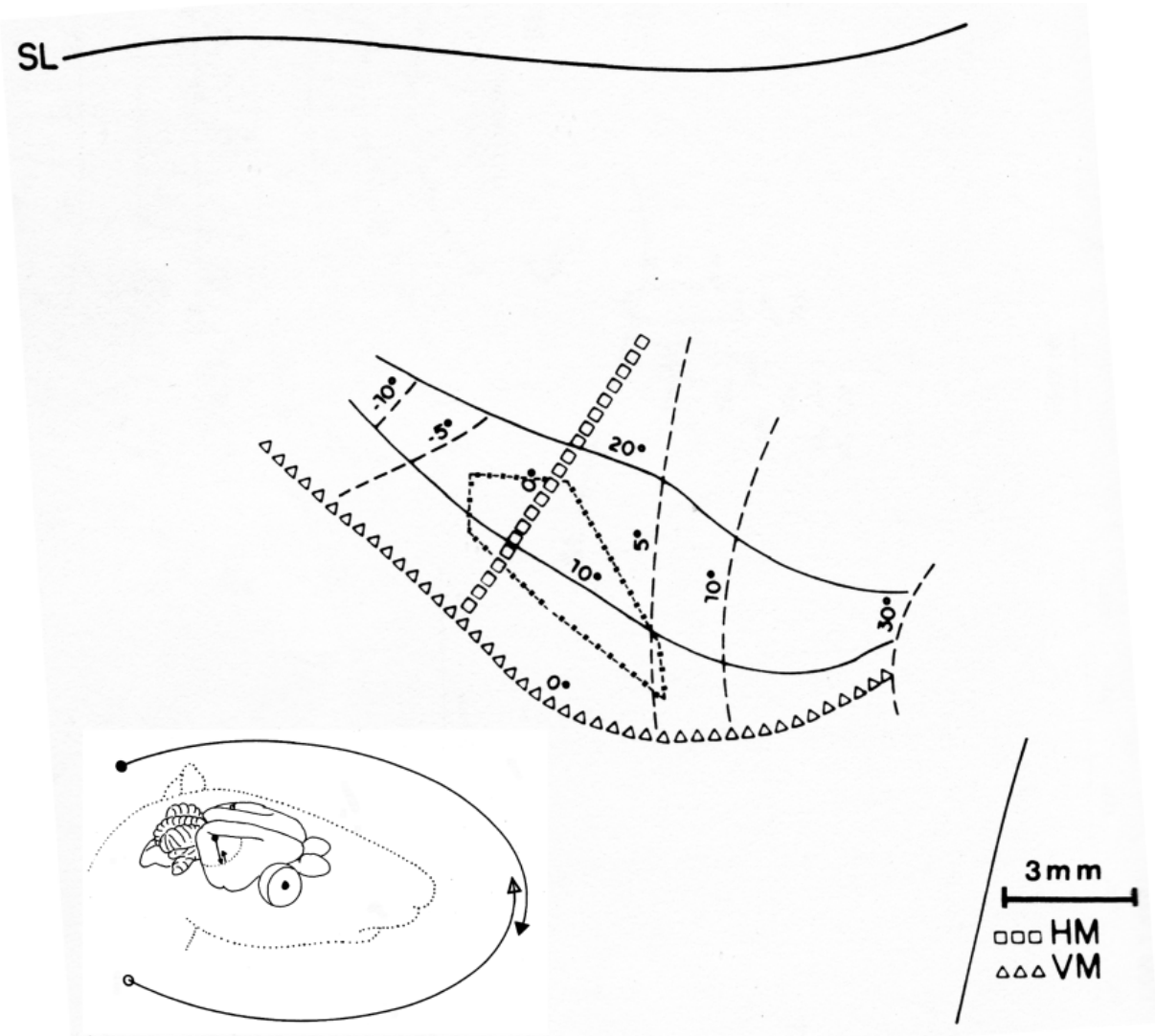

Figure 4. Schematic representation of the mono and binocular fields in the horizon and binocular multiunit response area in V1 (case Da850628). Arrows indicate schematic left and right monocular visual fields extent. The region of visual field where the arrows intersect (approximately $20 \mathrm{deg}$ ) indicates the binocular visual field. Binocular multiunit receptive fields were detected near the vertical meridian (dashed/dotted line). HM: horizontal meridian; VM: vertical meridian.

centralis (Hughes, 1977). Indeed rabbits (Hughes, 1971), goats (Hughes \& Whitteridge, 1973) and sheeps (Clarke \& Whitteridge, 1976) also align the visual streak to the horizon to increase resolution and motion detection. In these species even a casual observation at their heads suggests that the visual field of these animals area unusually large (Hughes, 1977; Picanço Diniz, 1987). In lateral eyed-species with well defined visual streaks it is common to find a mismatch between the ganglion cell densities along the horizon in the retina and the nasotemporal representation of the horizontal meridian in cortical map in V1 (Picanço-Diniz et al., 1992). Indeed a review of the published data confirms that all lateral-eyed mammals, with different life styles and retinal organizations, appear to exhibit similar mismatches between the retinal and cortical representation of the visual field. Moreover, these mismatches are larger in those species with well defined visual streaks such as rabbit and agouti (Hughes, 1977; Picanço-Diniz et al., 1992).

Representative examples of V1 maps and retinal ganglion cell isodensity lines adapted from elsewhere, including mouse (Drager \& Olsen, 1981; Wagor, Mangini, \& Pearlman, 1980), grey squirrel (Hall, Kaas, Killackey,\& Diamond, 1971; Hughes, 1977), hamster
(Tiao \& Blakemore, 1976 a,b), guinea-pig (Choudhury, 1978), agouti (Picanco-Diniz et al., 1991)and rabbit (Hughes, 1971) illustrate this point. Two of those species belong to Hystrichomorpha (agouti and guinea-pig), three belong to Myomorpha (rat, hamster and mouse) and one to the Sciurumorpha sub-order (grey squirrel). Among the Hystrichomorpha the agouti present a well defined visual streak with longer isodensity lines parallel to horizon and high centro-peripheral gradient of ganglion cell density in the retina (Silveira et al., 1989) whereas the guinea-pig present a rudimentary visual streak with low centro-peripheral gradient of ganglion cell density (Choudhury, 1978) (Figure 5).

In both rat (Fukuda, 1977) and hamster (Tiao \& Blakemore, 1976b) round ganglion cell isodensity lines were described suggesting the presence of area centralis. However in those species with round ganglion cells isodensity lines, the highest values of the cortical magnification in V1 maps were detected in the interception of the vertical and horizontal meridian, which does not coincide with the representation of the area centralis.

Figure 6 present detailed V1 maps (Wagor et al., 1980) and ganglion cell topography (round isodensity lines and area centralis (Drager \& Olsen, 1981)) for 

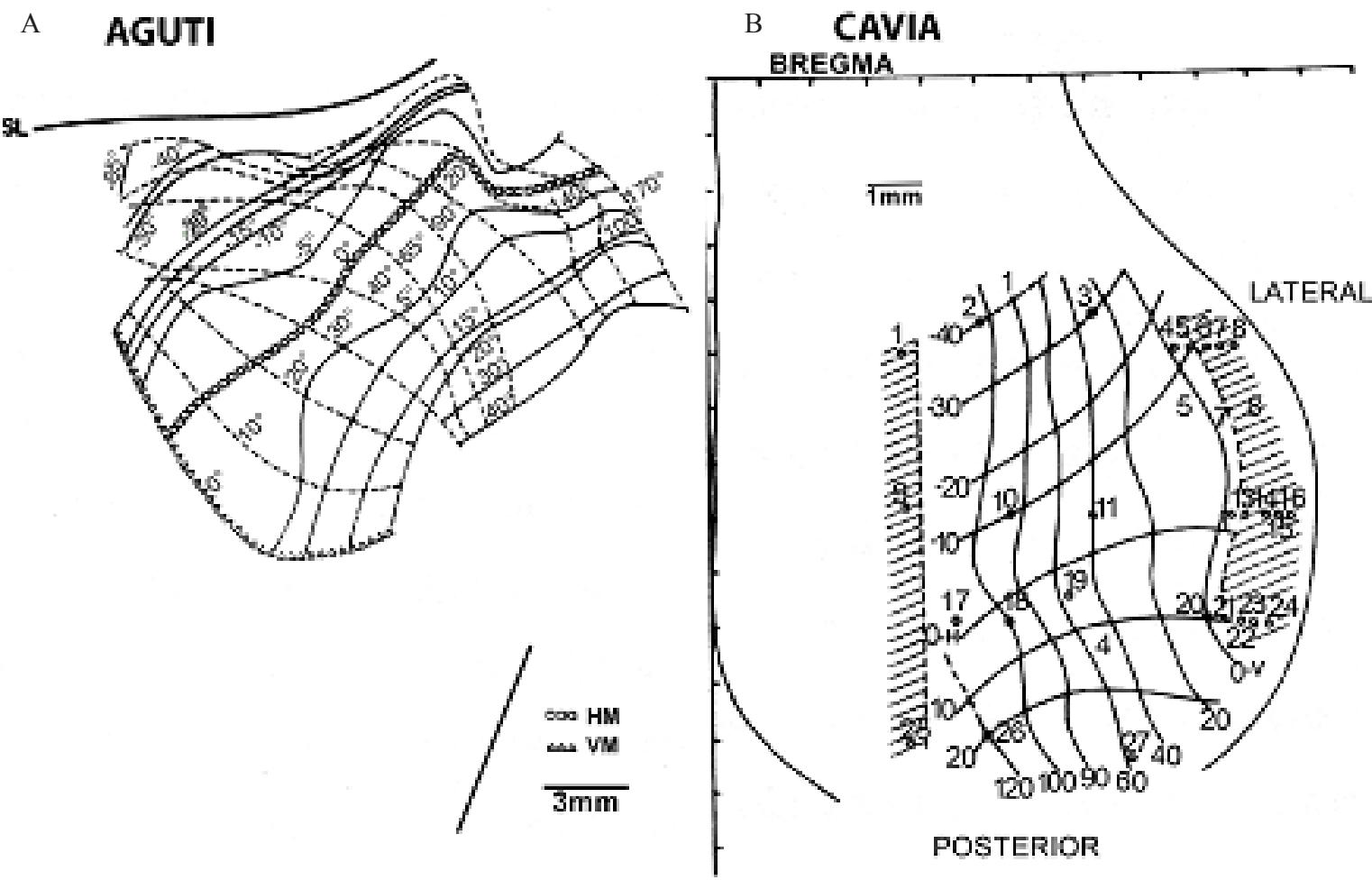

A'

B' CAVIA

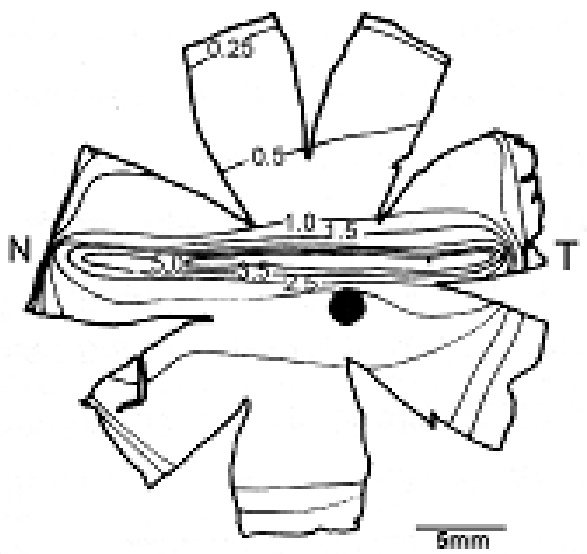

SILVEIRA (1985)

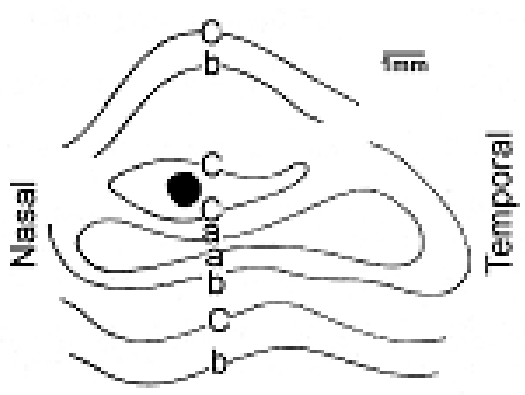

CHOUDHURY (1978)

Figure 5. Ganglion cells isodensity lines in the retina and V1 visual field representation in agouti (A, A'; modified from (Picanço Diniz 1987) and (Silveira 1985)) and guinea pig (B, B'; modified from (Choudhury 1978)). Isodensity lines (indicated by numbers or letters ) correspond to the distribution of retinal ganglion cells $\times 10^{3}$ and $\times 10^{2}$ in the agouti and guinea pig respectively. Note the nasotemporal asymmetries of V1 maps that do not match the temporo-nasal distribution of retinal ganglion cells. T: temporal; N: Nasal.

the mouse and grey squirrel (elongated isodensity lines and visual streak; (Hall et al., 1971; Hughes, 1977). Similar to the rat and hamster the highest values of the cortical magnification in V1 maps were detected in the interception of the vertical and horizontal meridian, which does not coincide with the representation of the area centralis. However, note a greater mismatch between the nasotemporal cortical magnification and retinal ganglion cell distribution in the grey squirrel as compared to the mouse due to the streak-like isodensity lines in the retina of the grey squirrel.

Figure 7 illustrates detailed V1 maps and retinal ganglion cell topography for hamster (Tiao \& Blakemore, 1976 a,b) and rabbit (Hughes, 1971). Other the even 
A

\section{MUS}

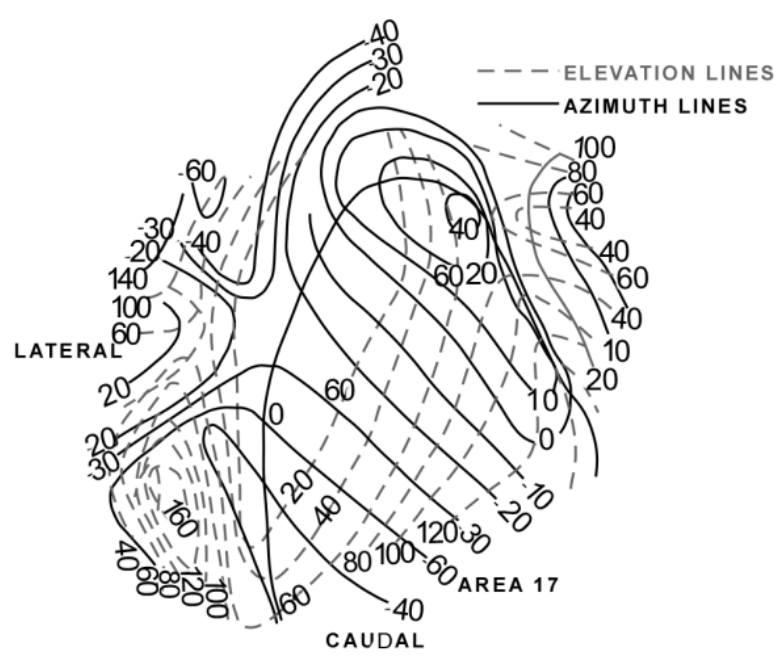

WAGOR et al (1980)

$\mathrm{A}^{\prime}$

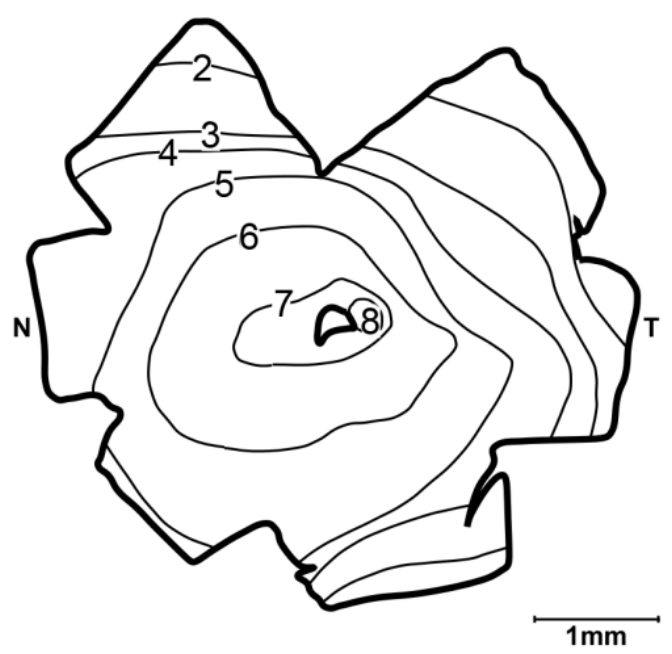

DRÄGER \& OLSEN (1981)
$\mathrm{B}$

\section{SCIURUS}

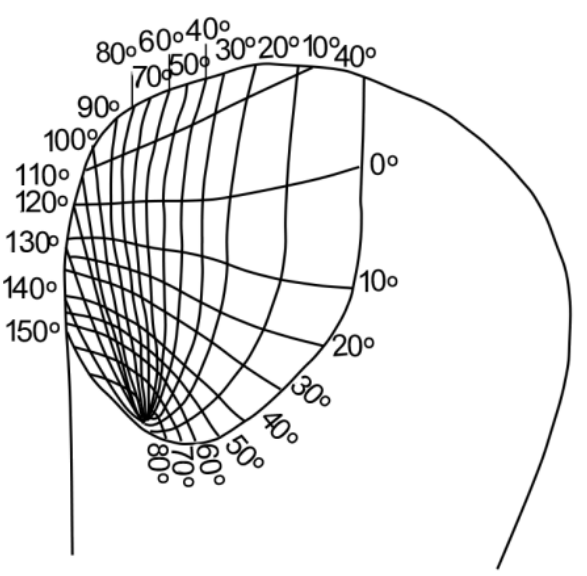

HALL et al (1971)

$\mathrm{B}^{\prime}$

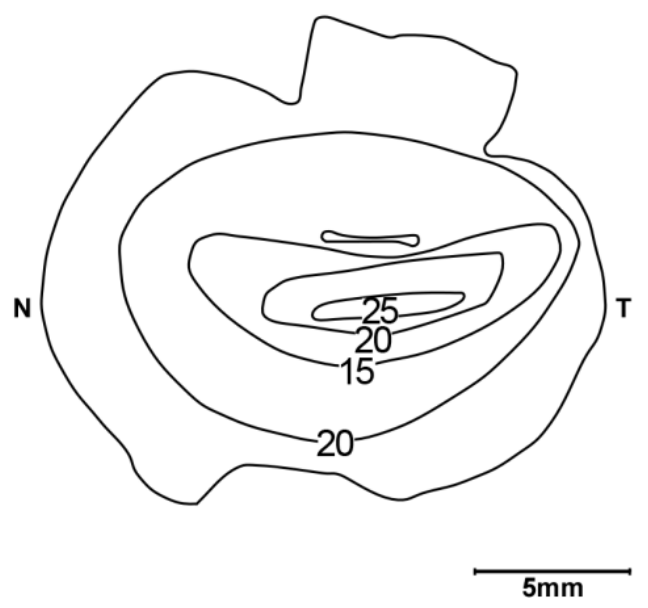

HUGHES (1977)

Figure 6. Ganglion cells isodensity lines in the retina and V1 visual field representation in mouse (A, A'; modified from Wagor et al., 1980 e Dräger \& Olsen, 1981) and grey squirrel (B, B'; modified from Hall et al., 1971 and Hughes, 1977). Isodensity line numbers indicate the distribution of ganglion cells $\mathrm{x} 10^{3}$. Note that the nasotemporal asymmetries of V1 maps do not match the temporo-nasal distribution of retinal ganglion cells. T: temporal; N: Nasal.

greater mismatch between ganglion cell topography and cortical magnification factor in the rabbit due to the defined visual streak.

Physiological implications of the nasotemporal asymmetries of the visual streak representation in agouti V1 cortical area.

In the agouti as much as $170^{\circ}$ of the visual field in the horizon of each eye (Picanço-Diniz, 1987) projects onto the major feature of the agouti retina, the visual streak, the extended area of high density of ganglion cells along the nasotemporal dimension of the horizontal meridian (Silveira, 1985; Silveira, Picanço-Diniz, \& Oswaldo-Cruz, 1989). The cortical representation of the contralateral visual streak in V1 is asymmetric in the agouti brain, with greater emphasis of the nasal region when compared to the central and temporal visual streak (Picanco-Diniz et al., 1991). In fact the representation of the nasal end of the visual streak defined by the latitudes and longitudes $\pm 10^{\circ}$ occupies ten times more cortical area than the central (latitude: $\pm 10^{\circ}$, longitude $68 \pm$ $10^{\circ}$ ) or temporal (latitude: $\pm 10^{\circ}$, longitude $170 \pm 10^{\circ}$ ) 
A

HAMSTER

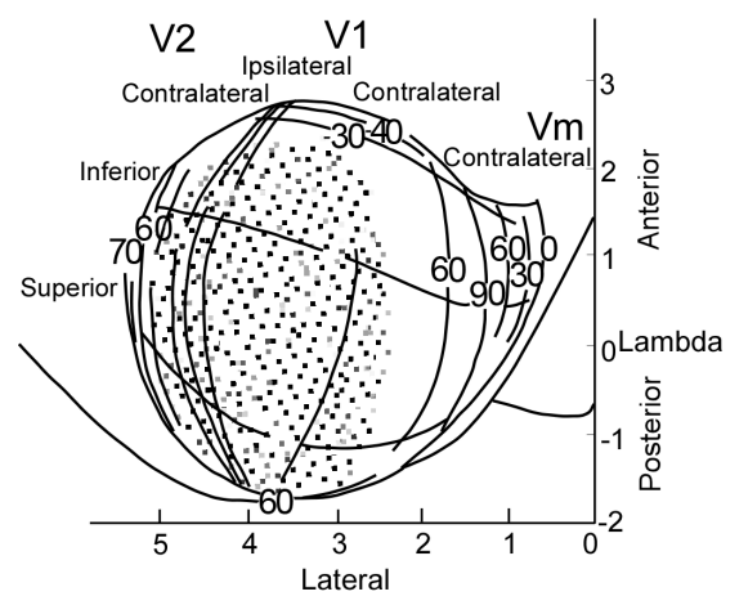

TIAO \& BLAKEMORE (1976 a)

$\mathrm{A}^{\prime}$

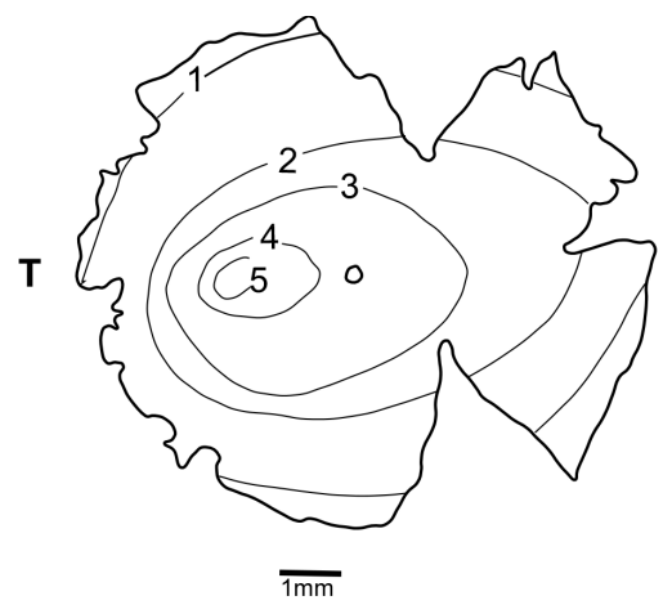

TIAO \& BLAKEMORE (1976 b)
B

\section{RABBIT}
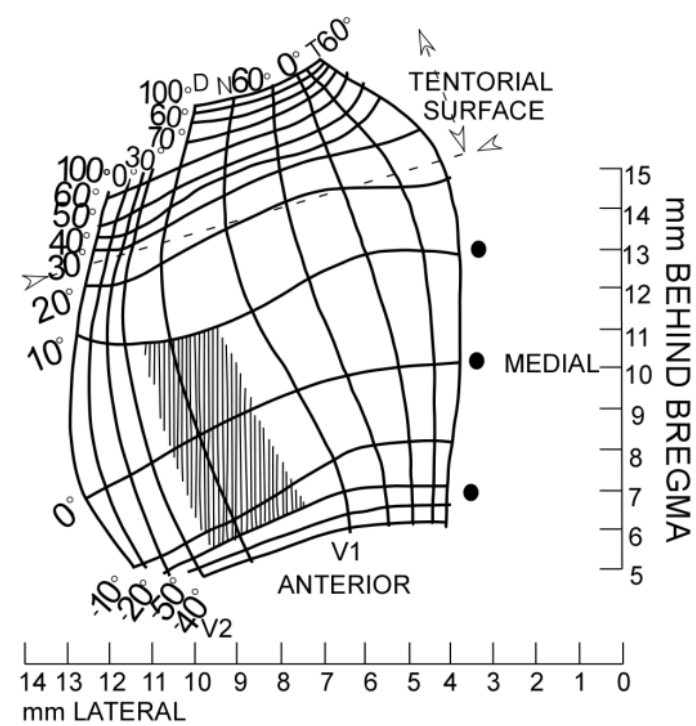

B'

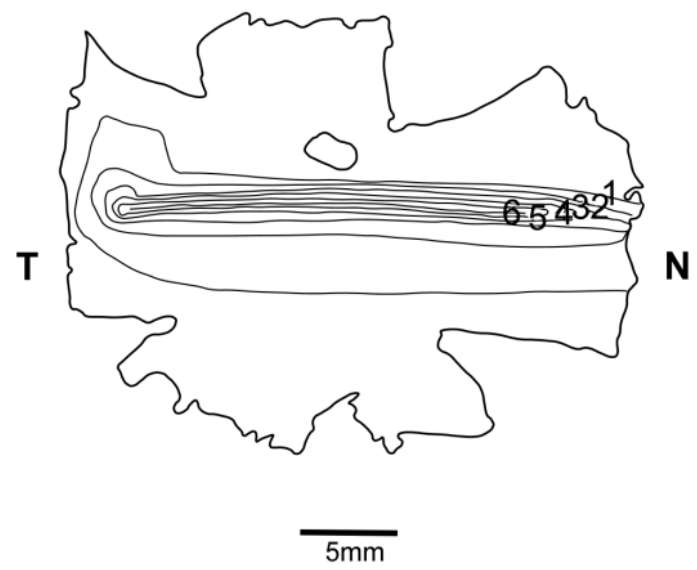

HUGHES (1971)

Figure 7. Ganglion cells isodensity lines in the retina and V1 visual field representation in hamster (A, A'; modified from Tião \& Blakemore, 1976a, b.) and rabbit (B, B'; modified from Hughes, 1971). Isodensity line numbers indicate the distribution of ganglion cells $\times 10^{3}$. Binocular field is indicated by hatched area. Once again the nasotemporal asymmetries of V1 maps do not match the temporo-nasal distribution of retinal ganglion cells. T: temporal; N: Nasal.

visual streak (Picanço-Diniz et al., 1992), whereas the ganglion cell density in the retina decreases towards the nasal and temporal visual fields.

This mismatch between ganglion cell density and the cortical magnification factor is inconsistent with the theory proposed by (Woolsey, Marshall, \& Bard, 1942) and largely accepted that the cortical representation in primary cortical areas is directly proportional to the density of peripheral innervations. In the visual system this rule is applied to the ganglion cell density.

We can think of at least two explanations of the mismatch between density of ganglion cells in the visual streak in the retina and cortical representation along the horizontal meridian in $\mathrm{V} 1$ of the rabbit and agouti, including intrinsic cortical factors and/ or retinal specializations (Choudhury, 1988; Hughes, 1977; Picanco-Diniz et al., 1991; Picanço-Diniz et al., 1992). In agreement with the first hypothesis it has been proposed that the distribution of non-pyramidal neurons of the pigmented rabbit primary visual cortex matches the nasotempoaral variation of the cortical magnification factor in V1 (Choudhury, 1988). On the other hand the occurrence of possible retinal specializations in the temporal ends of the visual streaks of agouti (Silveira, 1985; Picanço-Diniz et al., 1992) and rabbit (Provis, 1979) has not been confirmed in the 
rabbit (Oyster, Takahashi, \& Hurst, 1981) or seems to be not enough to explain the magnitude of the mismatch in the agouti. Since previous mapping studies on the rabbit superior colliculus (Hughes, 1971) revealed the absence of such mismatches the next candidates to explain the asymmetries could be the occurrence of possible specializations in the projections from LGN to V1 and/or intrinsic cortical mechanisms. Indeed, we have recently demonstrated differences in the density and dendritic trees of interneurons along the horizontal meridian in V1 of the agouti, with higher neuronal density and larger neuronal size near the vertical meridian in lateral V1. More branched dendritic trees were observed in the nasal representation as compared with the temporal periphery (unpublished observations).

In conclusion there is not a simple relationship between ganglion cell densities and the cortical magnification factor in the primary visual cortex of lateral-eyed mammals and cortical specializations may contribute to the intrinsic cortical mechanisms associated to the asymmetric magnification of the horizon in V1.

\section{Acknowledgments}

This project was sponsored by Brazilian Government research funds. Grant sponsor: Brazilian Research Council - CNPq and FINEP, CWPD is supported by Instituto Brasileiro de Neurociências - IBNnet and by Instituto Nacional de Febres Hemorrágicas Virais-CNPq

\section{References}

Choudhury, B.P. (1978). Retinotopic organization of the guinea pig's visual cortex. Brain Research, 144, 19-29.

Choudhury, B.P. (1988). Distribution of neurons in the pigmented rabbit's visual cortex. Experimental Neurology, 101, 458-463.

Clarke, P.G., \& Whitteridge, D. (1976). The cortical visual areas of the sheep. The Journal of physiology, 256, 497-508.

Drager, U.C., \& Olsen, J.F. (1981). Ganglion cell distribution in the retina of the mouse. Investigative ophthalmology \& visual science, 20, 285-293.

Fukuda, Y. (1977). A three-group classification of rat retinal ganglion cells: histological and physiological studies. Brain Research, 119, 327-334.
Hall, W.C, Kaas, J.H, Killackey, H., \& Diamond, I.T. (1971). Cortical visual areas in the grey squirrel (Sciurus carolinesis): a correlation between cortical evoked potential maps and architectonic subdivisions. Journal Neurophysiology, 34, 437-452.

Hughes A. (1971). Topographical relationships between the anatomy and physiology of the rabbit visual system. Documenta Ophthalmologica, 30, 33-159.

Hughes A. (1977). T The topography of vision in mammals of contrasting life styles: Comparative optics and retinal organization. In F. Cresctelli (Ed.), Handbook of Sensory Physiology(p. 813). Berlin:Springer-Verlag.

Hughes, A., \& Whitteridge, D. (1973). The receptive fields and topographical organization of goat retinal ganglion cells. Vision Research, 13, 1101-1114.

Oyster, C.W., Takahashi, E.S., \& Hurst, D.C. (1981). Density, soma size, and regional distribution of rabbit retinal ganglion cells. The Journal of Neuroscience 1, 1331-1346.

Picanço- Diniz, C. (1987). Organização do Sistema Visual de Roedores da Amazônia: Topografia das Áreas Visuais Corticais da Cutia. In Instituto de Ciências Biológicas, Laboratório de Neurofisiologia Eduardo Oswaldo-Cruz, pp. 297. Universidade Federal do Pará, Belém - Pará - Brasil.

Picanco-Diniz, C.W., Silveira, L.C., de Carvalho, M.S. \& OswaldoCruz, E. (1991). Contralateral visual field representation in area 17 of the cerebral cortex of the agouti: a comparison between the cortical magnification factor and retinal ganglion cell distribution. Neuroscience, 44, 325-333.

Picanço-Diniz, C.W., Silveira, L. \& Oswaldo-Cruz, E. (1992). A Comparative Survey of Magnification Factor in V1 and Retinal Ganglion Cell Topography of Lateral-Eyed MammalsEnglish. In R. Lent.(ed), The visual system from genesis to maturity (pp. 187198). Boston: Birkhäuser.

Provis, J.M. (1979). The distribution and size of ganglion cells in the regina of the pigmented rabbit: a quantitative analysis. The Journal of Comparative Neurology, 185, 121-137.

Silveira, L.C. (1985). Organização do Sistema Visual de Roedores da Amazôna: Óptica Ocular e Distribuição das C'[elulas Ganglionares Retinianas. . In Instituto de Ciências Biológicas, pp. 427. Universidade Federal do Pará, Belém, Pará - Brazil.

Silveira, L.C, Picanco-Diniz, C.W., \& Oswaldo-Cruz, E. (1989). Distribution and size of ganglion cells in the retinae of large Amazon rodents. Visual neuroscience 2, 221-235.

Tiao, Y.C., \& Blakemore, C. (1976a). Functional organization in the visual cortex of the golden hamster. The Journal of Comparative Neurology, 168, 459-481.

Tiao, Y.C., \& Blakemore, C. (1976b). Regional specialization in the golden hamster's retina. The Journal of Comparative Neurology, 168, 439-457.

Wagor, E., Mangini, N.J., \& Pearlman, A.L. (1980). Retinotopic organization of striate and extrastriate visual cortex in the mouse. The Journal of Comparative Neurology, 193, 187-202.

Woolsey, C., Marshall, W., \& Bard, P. (1942). Representation of cutaneous tactile sensibility in the cerebral cortex of the monkey as indicated by evoked potentials. Johns Hopkins Hospital Bulletin 70, 399-441. 